\title{
Supporting Information for Au Nanoparticle Enhancement of Plasma-Driven Methane Conversion into Higher Order Hydrocarbons via Hot Electrons
}

\author{
Bofan Zhao ${ }^{2}$, Indu Aravind ${ }^{1}$, Sisi Yang ${ }^{1}$, Yu Wang ${ }^{3}$, Ruoxi Li ${ }^{3}$, and Stephen B. Cronin ${ }^{* 1,2,4}$ \\ ${ }^{1}$ Department of Physics and Astronomy, ${ }^{2}$ Ming Hsieh Department of Electrical Engineering, \\ ${ }^{3}$ Mork Family Department of Chemical Engineering and Materials Science \\ ${ }^{4}$ Department of Chemistry
}

University of Southern California, Los Angeles, CA 90089, USA

*Corresponding author email: scronin@ usc.edu
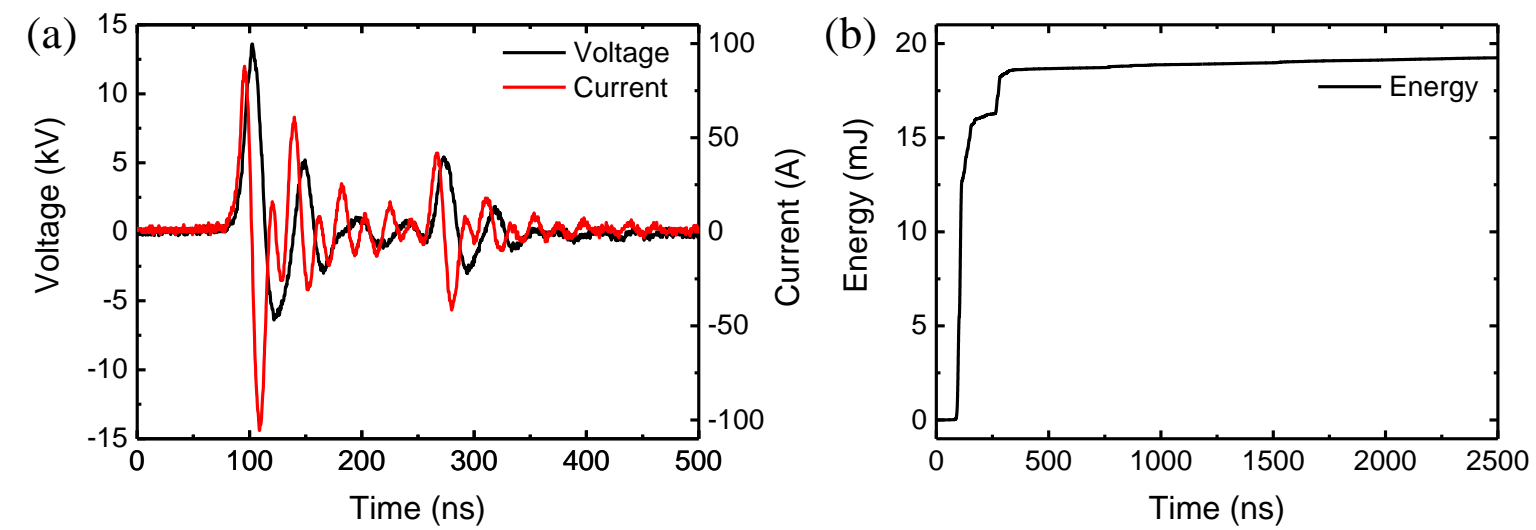

Figure S1. Example waveforms (vs. time) of (a) voltage and current, (b) energy of a 30X TPS high voltage pulse generator with the glass slide-based reactor at $400 \mathrm{~Hz}$ and $13 \mathrm{kV}$ power output.

Geometrical information of nanoparticles were extracted from HRTEM image (Figure 2c) and we used COMSOL Multiphysics simulation package with its material models and the electric current physics AC/DC module for electrostatic simulations with total simulation volume of $1250 \times 1000$ $\mathrm{x} 800 \mathrm{~nm}$. As shown in the schematic (Figure S1), we performed our simulation on a nanoparticle film, surrounded in air, with $550 \mathrm{~nm} \times 550 \mathrm{~nm}$ area and $5 \mathrm{~nm}$ thickness and two electrodes, separated for $750 \mathrm{~nm}$, were placed in-plane with the nanoparticle film. We monitored the electrical field 
distribution across the nanoparticle film under an applied DC voltage. We assumed that field cannot penetrate into nanoparticles, given the fact that at metallic losses are nearly negligible at low frequency region. After calculation with floating potential boundary condition, we get the field enhancement near surface which is defined by electric field intensity with nanoparticles over its counterpart without nanoparticles.

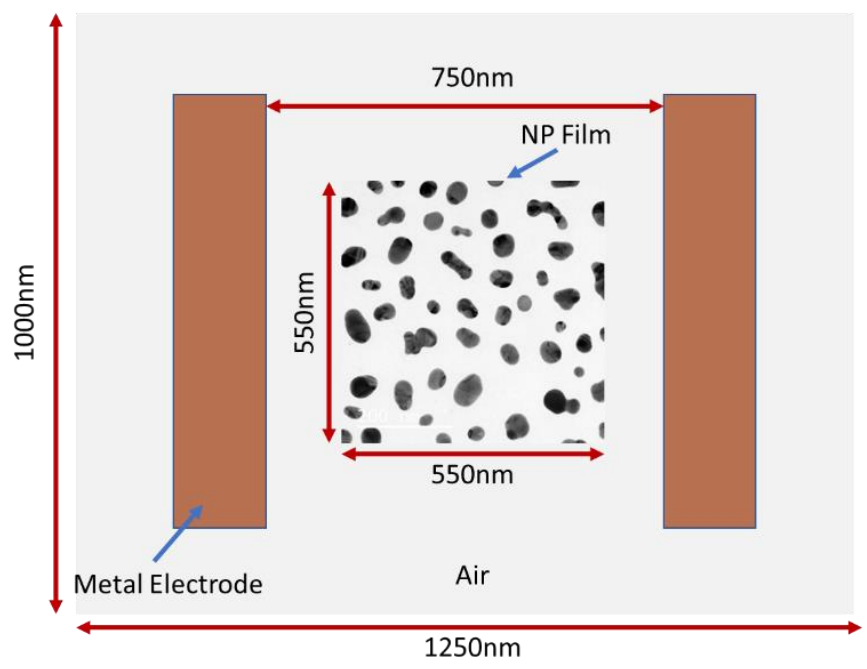

Figure S2. A schematic diagram of the structure of our simulated sample. 


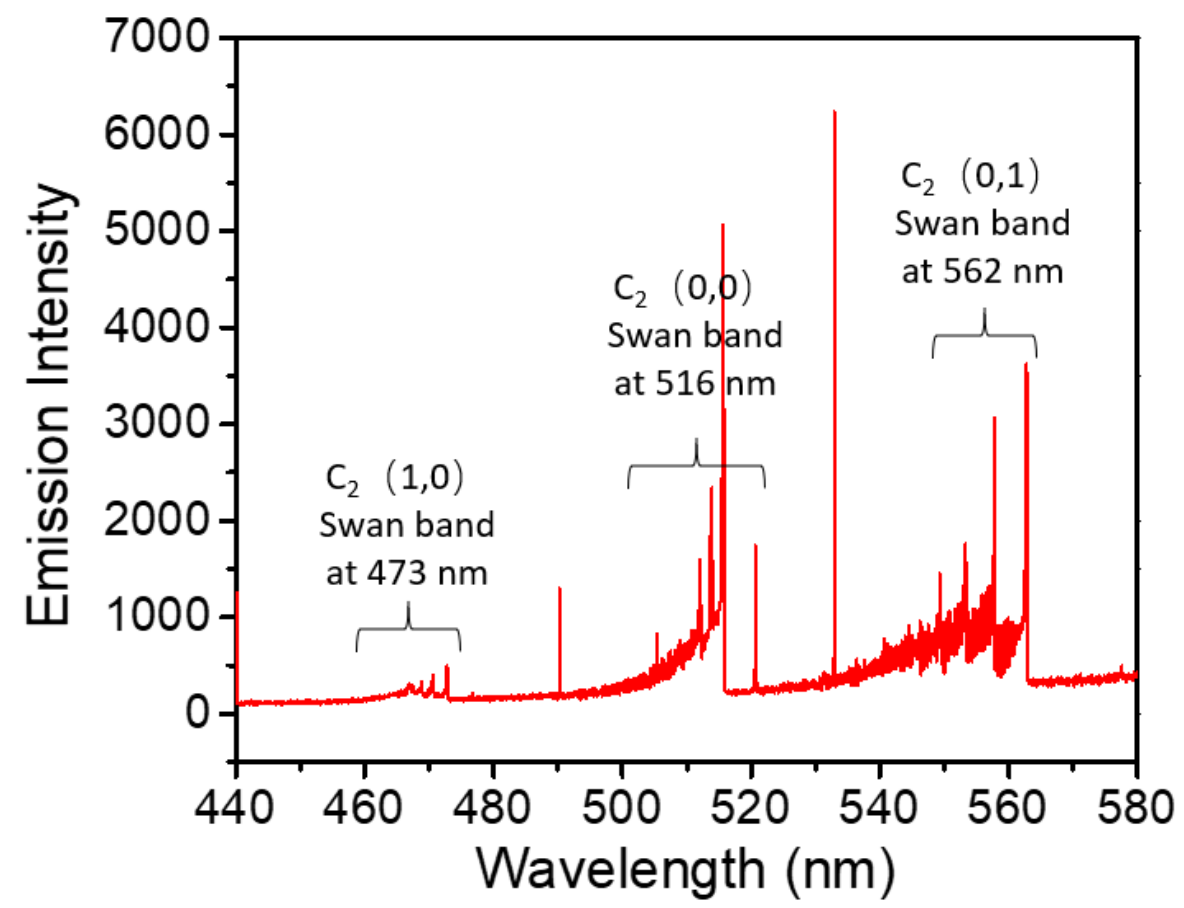

Figure S3. Emission spectrum exhibiting Swan bands obtained during plasma discharge. 


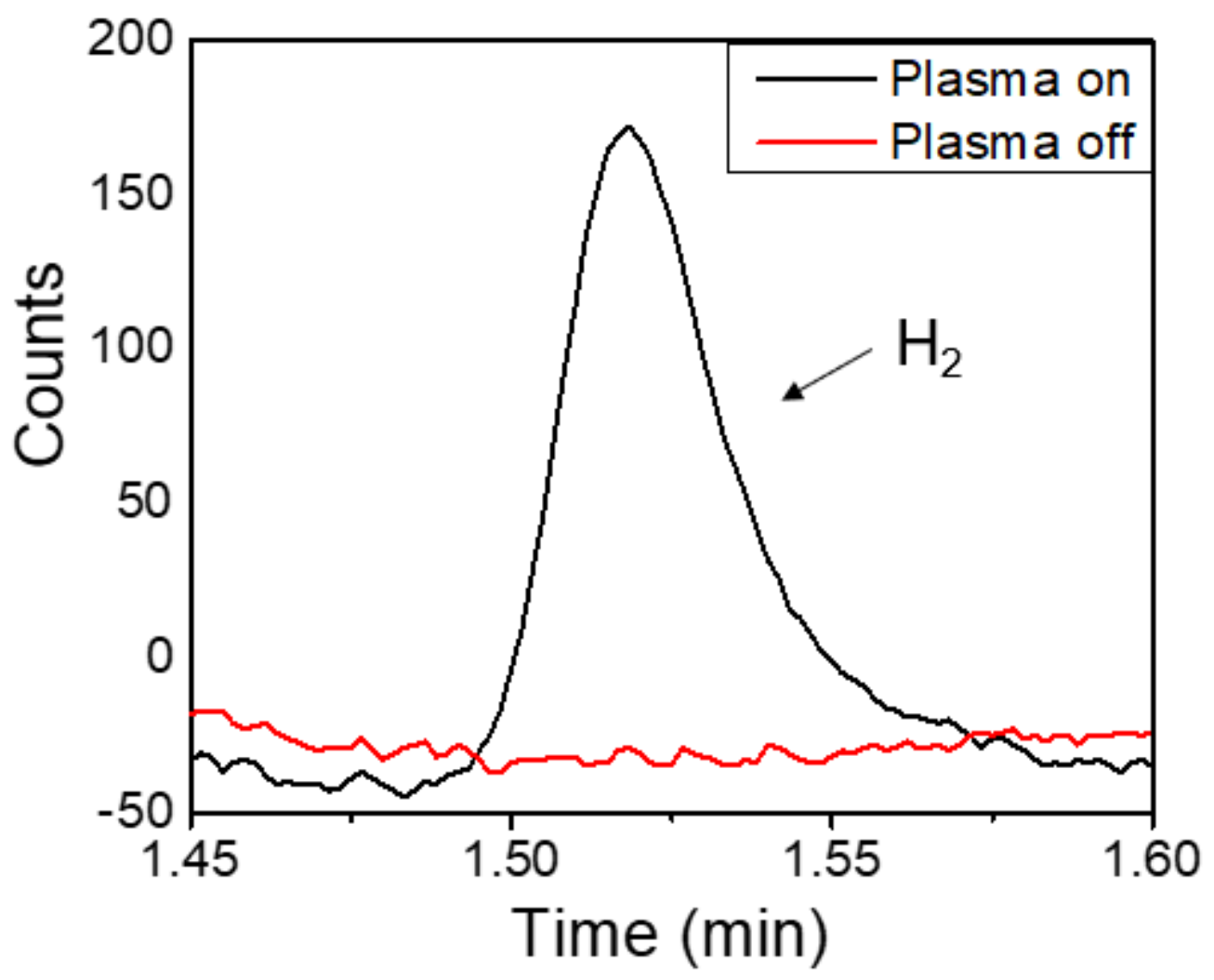

Figure S4. Gas chromatography result showing $\mathrm{H} 2$ produced during plasma discharge in the coaxial reactor. 


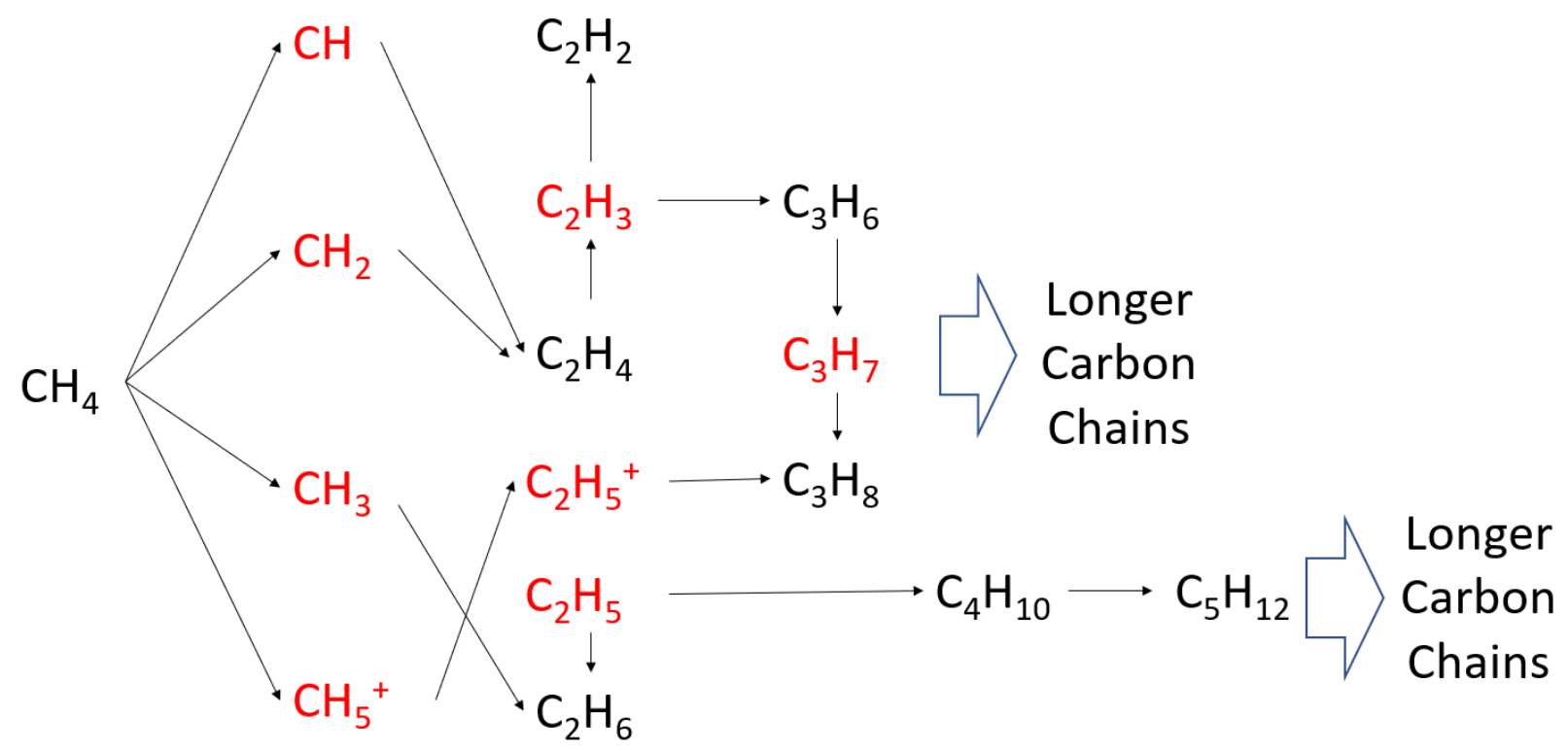

Figure S5. Summary of potential reaction pathways of methane upconversion (black molecules represent stable products and red molecules are unstable intermediates). 